\title{
On the Applicability of an Unsteady Aerodynamic ROM to the Transonic
}

\section{Flight Regime}

\author{
Torstens Skujins * \\ Carlos E. S. Cesnik ${ }^{\dagger}$ \\ University of Michigan, Ann Arbor, Michigan 48109
}

\begin{abstract}
Accurate prediction of aerodynamic loads throughout multiple flight regimes is vital for the development of control algorithms for vehicles which pass through different regimes during the course of a flight. As such, a reducedorder modeling methodology based on linear convolution combined with a nonlinear correction factor initially developed for hypersonic flight has been extended to the transonic flight regime. Errors mostly remained small when the unsteady lift, drag and moment coefficient results were compared with direct CFD simulations over a range of oscillation frequencies and amplitudes, though the drag coefficient did show larger errors at higher oscillation frequencies. A methodology to determine the optimal number and location in the parameter space of the ROM construction sampling points has also been developed. Results showed good agreement between the coefficients calculated by this method and those calculated by direct CFD simulation.
\end{abstract}

\section{Introduction}

Accurate calculation of unsteady aerodynamic loads due to vehicle rigid body pitch and plunge motion as well as elastic deformations is vital to both the development of control algorithms and flutter boundary prediction. Often times, vehicles will encounter several different flight regimes throughout the duration of a single flight; for example, hypersonic vehicles must pass through the subsonic, transonic, and supersonic regimes on the way to hypersonic flight. The different flight regimes offer distinct challenges when modeling the unsteady aerodynamic loads. In the transonic regime, moving shocks and other effects result in a nonlinear flow field, thus complicating the prediction of aerodynamic forces. In the hypersonic regime, strong shocks and the relatively large magnitude of the aerodynamic loads are among the factors which present further modeling challenges. When modeling the unsteady aerodynamic loads on a single vehicle throughout several different flight regimes, a single mathematical form representing the loads in all regimes is important to have when coupling with a full aerothermoelastic framework, as the efficiency is increased if the same aeroelastic equations can be used without modification regardless of the flight regime in question.

Computational fluid dynamics (CFD)-based reduced-order models (ROMs) provide an effective way to model unsteady aerodynamic loads. Once constructed, the models run orders of magnitude faster than full CFD solutions while preserving a high level of the accuracy seen by the computational simulations. Silva ${ }^{1}$ developed a method using first and second-order Volterra kernels to predict the unsteady loads on a transonic airfoil; similar methodology was then extended to create a state-space system useful for aeroservoelastic analysis. $^{2}$ Raveh $^{3}$ uses step responses to find the generalized aerodynamic forces for aeroelastic analysis in the transonic regime. One major drawback of many reduced-order models is that they are only valid for flight conditions immediately around those from which the model was constructed. Efforts have been made to make the ROMs valid over a range of parameters. Glaz et al. ${ }^{4}$ use an unsteady surrogate-based approach to construct a model for unsteady rotorcraft dynamics over a range of pitch/plunge motions and Mach

\footnotetext{
*Graduate Research Assistant, University of Michigan, Email: tskujins@umich.edu, Member AIAA.

${ }^{\dagger}$ Professor, Department of Aerospace Engineering, University of Michigan, Email: cesnik@umich.edu, 1320 Beal Avenue, 3024 FXB, Ann Arbor, MI 48109-2140, Ph. (734) 764-3397, Fax: (734) 764-0578, AIAA Associate Fellow.
} 
numbers. Silva ${ }^{5}$ uses a convolution-type of methodology to construct a state-space ROM which is then used over a range of velocities in the transonic regime by modifying the time step of the numerical integration. Other efforts for parameter-independent ROMs have focused on the analysis of flight test data. Lind et al. ${ }^{6}$ create velocity-independent kernels by using curve fits of flight test data gathered at different conditions. Baldelli et al. ${ }^{7}$ create a model valid over a range of dynamic pressures by combining linear and nonlinear operators for model construction. Prazenica et al. ${ }^{8}$ extrapolate kernels found at different flight conditions to create one model valid over a range of conditions. Omran and Newman ${ }^{9}$ use Volterra series submodels in different domains, such as pre-stall and post-stall, to construct an overall global piecewise Volterra series model.

The above ROM efforts have tended to focus on the transonic regime rather than the applicability across multiple flight regimes. However, the most efficient way to model the aerodynamics of a vehicle designed to fly in multiple regimes is to have a single ROM method applicable to each of the regimes while also minimizing the computational cost of model construction. Ref. 10 lays out the basic framework for a CFD-based ROM technique for the calculation of unsteady aerodynamic loads applied to the hypersonic flight regime. Rather than being valid only for the parameters at which it is constructed, the model is valid over a range of modal amplitudes and Mach numbers. Though it has only been tested at hypersonic flight conditions, nothing about the setup of the method inherently limits it to this specific regime or any specific vehicle geometry as well. The purpose of this paper is to assess the accuracy of the ROM applied to the transonic regime with the overall goal in mind to develop one mathematical ROM format applicable across multiple flight regimes. Additionally, a methodology is developed to determine beforehand the number CFD runs, as well as the various parameters for those runs, necessary for model construction; this improves the efficiency of model construction.

\section{ROM Methodology}

The reduced-order modeling methodology used here is a combination of linear convolution and a nonlinear correction factor. Linear convolution was chosen over other ROM methodologies due to the relative ease of implementation into the CFD code. Fig. 1 shows a diagram of the ROM framework. The ROM inputs are the structural mode shapes as well as the modal amplitudes of displacement at each time step. The structural mode shapes are used both to find the step responses of the system and to calculate the correction factor. All calculations pertaining to the structural mode shapes are calculated up front during initial model construction and thus do not need to be repeated during ROM simulations. Then, the modal amplitudes are convolved with the modal step responses to find the uncorrected ROM response. Finally, at each time step, the correction factor is applied to the uncorrected response to give the final, corrected ROM response.

\section{A. Convolution}

The response of a linear system to an arbitrary input can be found if the response of the system to a unit step $(H(t))$ or unit impulse $(h(t))$ function is known. The response $y(t)$ due to an arbitrary input $f(t)$ is found through the use of convolution: ${ }^{1,11}$

$$
y(t)=f(0) H(t)+\int_{0}^{t} \frac{d f}{d t}(\tau) H(t-\tau) d \tau
$$

Since the unit impulse is the derivative of the unit step, integration by parts yields

$$
y(t)=f(t) H(0)+\int_{0}^{t} f(\tau) h(t-\tau) d \tau
$$

Equations 1 and 2 are the two forms of Duhamel's integral. For this work, the unit step was chosen over the unit impulse due to improved CFD simulation results of the step over the impulse.

\section{B. Correction Factor}

The aerodynamics of the flight regimes considered in this work are not linear. Shock waves create strong, nonlinear discontinuities in the flow field which in general are not captured well through linear analysis, such as the the linear convolution described previously. For this reason, a nonlinear correction factor has been 


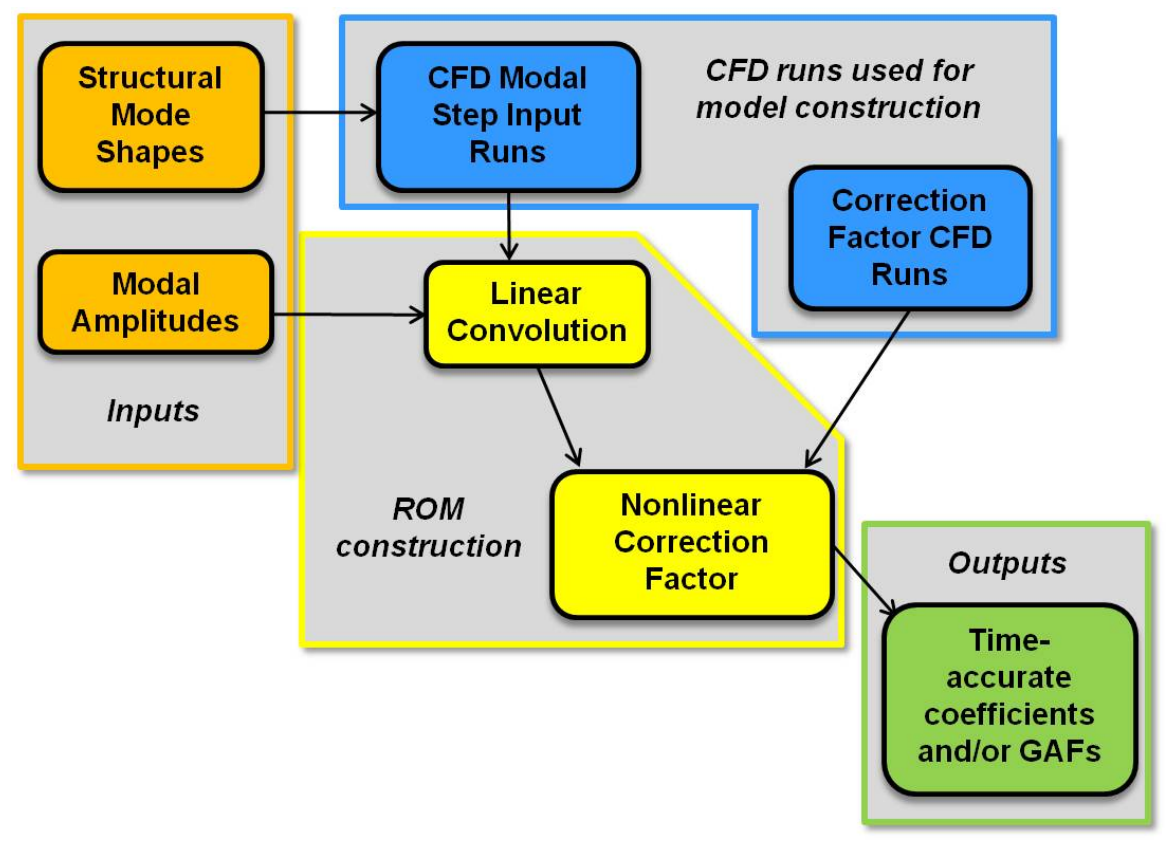

Figure 1. Overall ROM framework

developed. Consider a sample airfoil undergoing multi-modal oscillations. The multi-modal correction factor is defined as follows, here presented for simplicity as a two-mode excitation:

$$
f_{c}=\frac{y_{12}-\delta}{y_{1}+y_{2}-\delta}
$$

In Eq. 3, $y_{12}$ is the final quasi-steady response after steps of certain amplitudes have been simultaneously applied to each of the first two modes, $y_{1}$ and $y_{2}$ are the final quasi-steady responses of the individual steps of each of the modes, and $\delta$ is an offset introduced to prevent situations in which the denominator is zero and hence the correction factor would give an inaccurate result. To make the ROM applicable across a wide range of flight conditions, the correction factor value is calculated at points throughout the parameter space through CFD simulations; see Ref. 10 for the details of these runs. In this work, parameters which have been considered include modal amplitudes and Mach number. The sampling points in the parameter space are found through the use of nearly-orthogonal Latin hypercube sampling, ${ }^{12}$ which ensures an even distribution of points and thus avoids points clustering in certain areas. Next, a kriging surface is created with each of the parameters as variables. Kriging ${ }^{13,14}$ is a methodology which fits a surface to data generated by computational experiments, taking advantage of the fact that, unlike physical experiments, computational experiments lack random error. Then, to apply the correction factor, the motion of each of the modes is individually convolved with the step response and added together via superposition (the denominator in Eq. 3). Rearranging Eq. 3, the corrected response $y_{12}$ at a non-sampling point location in the parameter space is found by:

$$
y_{12}=f_{c}\left(y_{1}+y_{2}-\delta\right)+\delta
$$

where $y_{1}+y_{2}$ is the superimposed individual modal responses. At each time step, the correction factor corresponding to the specific modal amplitudes and Mach number is picked off the kriging surface and used in Eq. 4 to obtain the final corrected response. In this work, separate kriging surfaces are constructed for the correction factors pertaining to the lift, drag, and moment coefficients.

Though the correction factor requires sampling points to be calculated using CFD, the CFD runs are all conducted up front. Once the ROM has been constructed, no further code evaluations are necessary. Thus, after the initial computational expense for model construction, the ROM will run in a computationally cheap manner. 


\section{Basic Problem Definition and Setup}

The CFD code used in this study is CFL3Dv6, developed at NASA Langley. ${ }^{15}$ The code is capable of solving the Euler/Navier-Stokes equations for both steady and unsteady flows on two and three-dimensional structured grids and has mesh deformation capability. Modal inputs are given to the geometries by utilizing the code's mesh deformation capabilities. Response quantities tracked are the lift, drag, and moment coefficients. All solutions are Euler solutions.

To test the ROM's applicability to the transonic regime, a CFD grid of the AGARD 445.6 wing $^{16}$ has been obtained from NASA Langley, where wind tunnel aeroelastic tests have been conducted on the wing. The wing has also been widely used for computational aeroelastic studies. ${ }^{3,17-20}$ The grid is a structured $65 \times 193 \times 41$ grid with the $i$ direction being along the span, $j$ direction along the chord, and $k$ direction normal to the wing surface. Figure 2 shows the grid as well as a zoomed-in figure of the wing itself.

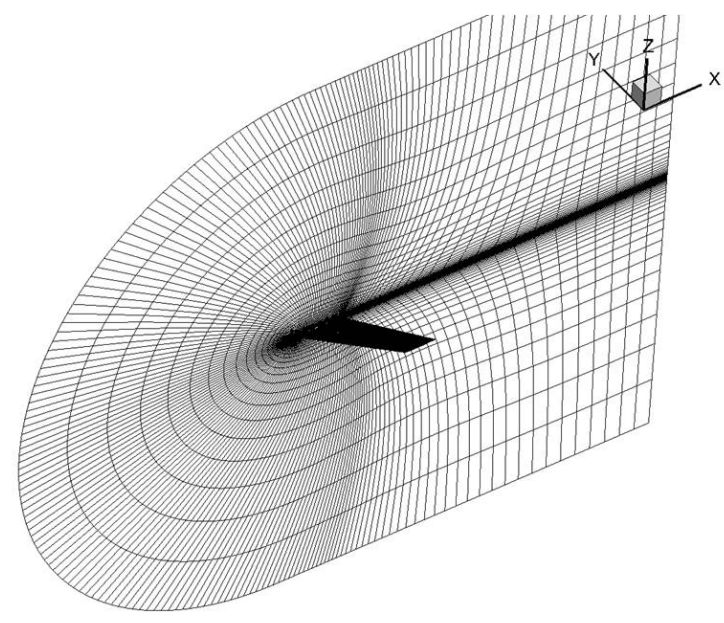

(a) AGARD grid

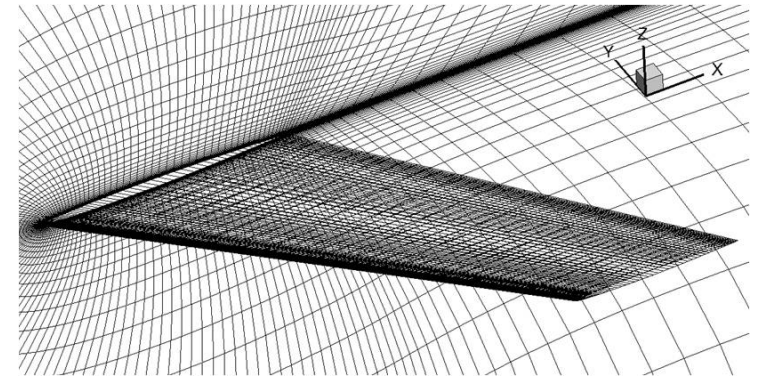

(b) AGARD wing close-up

Figure 2. AGARD 445.6 wing

Oscillations of the first three elastic mode shapes of the wing will be considered. These mode shapes, shown in Fig. 3, are the same that have been used in other studies as well. ${ }^{21,22}$ Note that, for each mode shape, the unit step input corresponds to a maximum wing deflection of just around $0.1 \%$ of the span.

\section{A. Parameter Space Sampling Point Determination}

As the number of parameters considered for the ROM increases, the number of sampling points in the parameter space necessary to allow the kriging surface to accurately represent the correction factor function will also increase with the added dimensionality. Thus, it is desirable to have a methodology to help determine beforehand the optimal number and location of the sampling points within the parameter space. Without any knowledge of this, too many or too few points may end up being used, resulting in either reduced surface accuracy or unnecessary computational expense. One requirement of this methodology is that it must be relatively computationally cheap. Otherwise, it would make more sense to use the "brute force" method and just keep using individual CFD runs to add more points to the surface.

The basic idea of the sampling point methodology is that, when the wing is in an elastically-deformed position, it can be approximated as a series of chordwise-rigid segments along the span which are at different angles of attack. Since the correction factor methodology discussed in Section II.B relies on the quasi-steady coefficients after a certain modal deformation has been input, the lift and drag at each of the chordwise segments along the span will be found utilizing steady rigid CFD simulations conducted at varying angles of attack and Mach numbers. While the individual segments will also undergo a plunge motion in addition to pitching motion during elastic deformations, these plunge motions are neglected here due to the quasisteadiness of the CFD solutions being found. The specific steps to the method are as follows: 

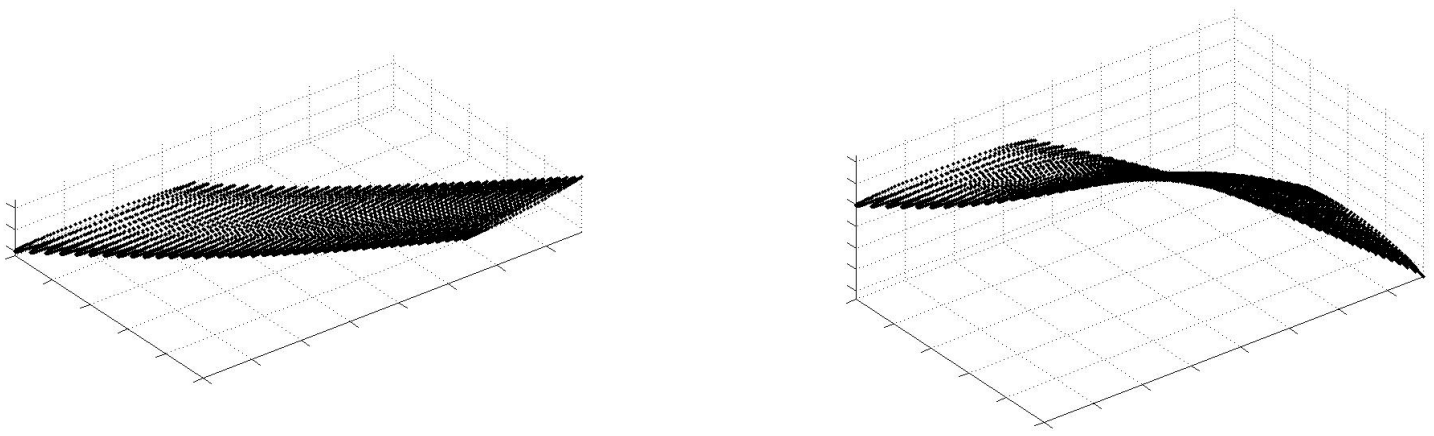

(a) Mode 1, $\omega_{1}=9.6 \mathrm{~Hz}$

(b) Mode 2, $\omega_{2}=38.2 \mathrm{~Hz}$

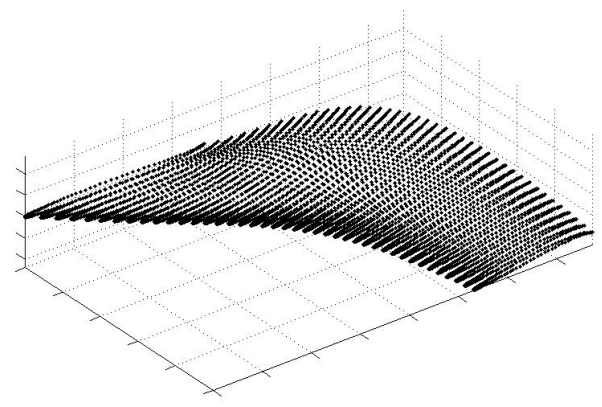

(c) Mode 3, $\omega_{3}=48.2 \mathrm{~Hz}$

Figure 3. AGARD 445.6 wing mode shapes 
1. Divide the wing into chordwise segments along the span. In this case, the AGARD wing has been divided into 11 segments.

2. Conduct steady, rigid CFD runs at various Mach numbers and angles of attack which have been determined by Latin hypercube sampling. The Latin hypercube dimensionality will remain at 2 (angle of attack and Mach number) regardless of how many modes are being considered. Thus, the total number of runs remains relatively low, and steady runs are computationally cheaper than unsteady runs.

3. Track the lift and drag forces on each of the chordwise segments, taking into consideration the spanwise width of each segment. Construct separate kriging surfaces for the lift and drag forces at each of the segments.

4. For a certain wing deformation, calculate the local angle of attack at each wing segment.

5. Pick the lift and drag forces off the kriging surfaces for each segment corresponding to the specific Mach number and local angle of attack; sum them together to find the lift and drag for the entire wing.

6. Calculate the coefficients for the wing. These values can then be used to formulate the correction factor for that particular set of overall modal input parameters and Mach number.

For the remainder of this paper, this methodology will be referred to as the method of segments.

\section{Error Metric}

The error metric used to assess the accuracy of the ROM is the normalized root mean squared error over all time steps of a run. The error $\epsilon$ at a particular time step $i$ is defined as follows:

$$
\epsilon_{i}=\frac{y_{C F D, i}-y_{R O M, i}}{C F D_{\max }-C F D_{\min }}
$$

In Eq. $5, y_{C F D, i}$ and $y_{R O M, i}$ are the CFD and ROM response values, respectively, at time step $i$, and $C F D_{\max }$ and $C F D_{\min }$ are the maximum and minimum CFD response values calculated throughout the entire test. Thus, the error metric can be defined as:

$$
\text { error }=\frac{1}{N} \sum_{i=1}^{N} \epsilon_{i}
$$

where $N$ is the total number of time steps in the run.

\section{Single Modal Oscillation Studies}

The first series of investigations consisted of characterizing the applicability of the ROM methodology to oscillations of the first mode only. Kriging surfaces for the correction factors pertaining to the lift, drag, and moment coefficients were constructed for parameter range shown in Table 1, where the amplitude is given in terms of multiples of the step input.

Table 1. Single modal oscillation parameter values

\begin{tabular}{ccc}
\hline \hline Parameter & Min & Max \\
\hline$M$ & 0.8 & 1.2 \\
Amplitude & $-100 \times$ & $100 \times$ \\
\hline \hline
\end{tabular}




\section{A. Kriging Surfaces}

Kriging surfaces, shown in Fig. 4, were constructed for the correction factors corresponding to each of the coefficients. Due to the relatively large gradients around Mach 1, separate surfaces were constructed for suband supersonic Mach numbers. Also, since the AGARD 445.6 airfoil is symmetric, negative amplitude lift and drag coefficient values used for correction factor surface construction were simply the opposite of and the the same as, respectively, those found for the corresponding positive amplitude, thus reducing the total number of CFD runs required.

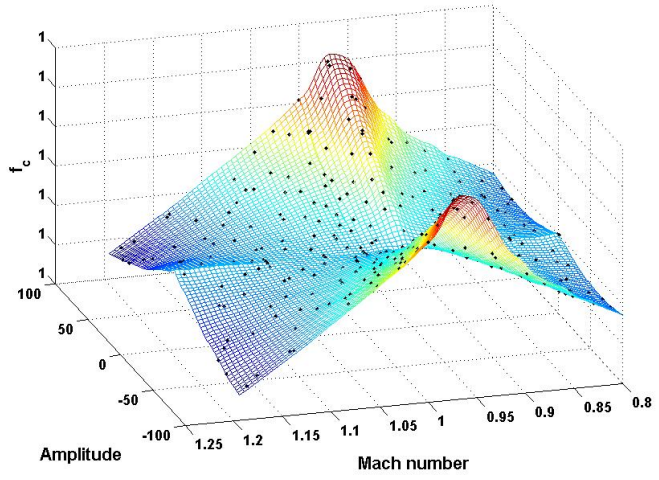

(a) $f_{c}$, lift coefficient

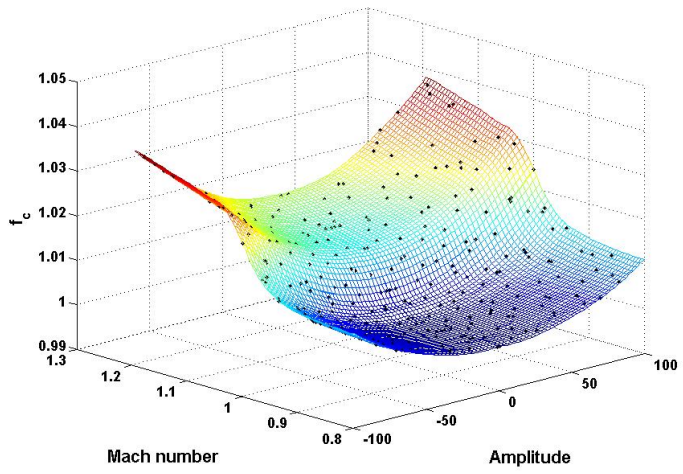

(b) $f_{c}$, drag coefficient



(c) $f_{c}$, moment coefficient

Figure 4. Kriging surfaces for mode 1

A total of 81 sampling points, found using Latin hypercube sampling of the parameter space, was used for the subsonic kriging surface, while 63 points were used for the supersonic surface. An offset value $(\delta)$ of $10^{6}$ was used. For an objective measure of the surface's ability to represent the correction factor function over the parameter space, the ratio of the surface's mean squared error (MSE) and variance were calculated throughout the parameter space. The MSE was calculated using a built-in MATLAB function, and details of the calculation methodology can be found in Refs. 13,14, and 23. Table 2 shows the ratio values for the correction factor corresponding to each of the coefficients for both the sub- and supersonic kriging surfaces. This ratio provides a user-defined stopping criterion for determining the number of points to be used for surface construction.

\section{B. Amplitude Tests}

To test the accuracy of the ROM as the amplitude of oscillation increases, sinusoidal oscillation test cases were generated with Mach number and oscillation frequency held constant while the maximum amplitude varied. These tests were repeated for both Mach 0.9 and Mach 1.1. For each test, the ROM was based on a step input generated at Mach 0.9, and the oscillation frequency is $\omega_{1}$. The ROM results at Mach 0.9 (solid 
Table 2. Kriging Surface maximum MSE/variance ratio values

\begin{tabular}{ccc}
\hline \hline Coefficient & Subsonic & Supersonic \\
\hline Lift & 0.0299 & 0.0058 \\
Drag & 0.0101 & 0.0106 \\
Moment & 0.0084 & 0.0023 \\
\hline \hline
\end{tabular}

lines) are compared to uncorrected ROM results (dashed lines), which consist of only linear convolution without any correction factor. Fig. 5 shows the errors corresponding to each test.

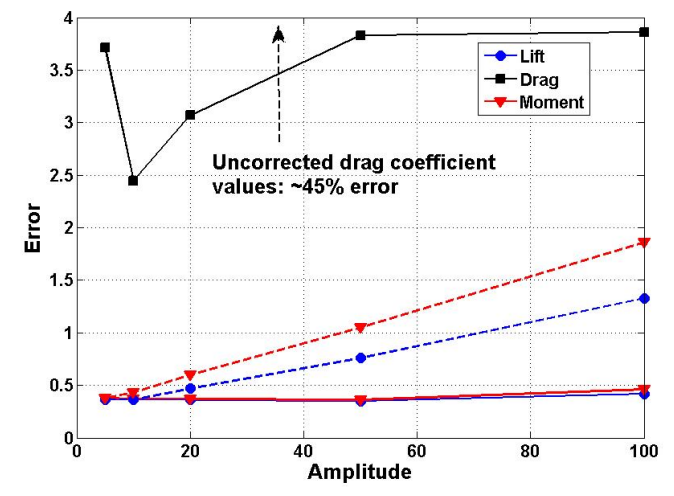

(a) Mach 0.9

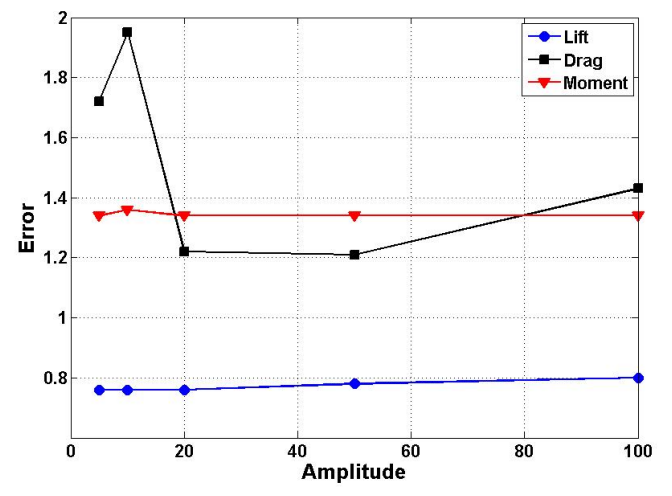

(b) Mach 1.1

Figure 5. Errors for increasing amplitude of oscillation

Fig. 5(a) shows that, while the correction factor ROM shows improved agreement with the CFD results for the lift and moment coefficients, the overall errors seen for both the corrected and uncorrected ROMs are relatively small. However, for the drag coefficient, the corrected ROM errors remain small over the range tested, while the uncorrected ROM errors are large, around 45\%. Fig. 6 shows the drag coefficient comparison between the corrected ROM, uncorrected ROM, and CFD results corresponding to amplitude 100 in Fig. 5(a). Qualitatively, the corrected ROM and CFD results match well, while the uncorrected ROM does not provide a close approximation at all.

\section{Frequency Tests}

To test the accuracy of the ROM as oscillation frequency increases, tests were conducted at constant Mach number and oscillation amplitude but with oscillation frequencies ranging from $\omega_{1}$ to $5 \omega_{1}$. As with the amplitude tests, these tests were repeated for both Mach 0.9 and Mach 1.1; oscillation amplitude was held constant and $20 \times$. The results are shown in Fig. 7 .

The results show that the errors do increase with oscillation frequency. This is likely due to the increased unsteadiness inherent with increased reduced frequencies combined with a quasi-steady correction factor formulation. For a more qualitative comparison, Fig. 8 shows the lift and drag comparisons for the test case corresponding to a frequency of $4 \omega_{1}$ at Mach 0.9.

Fig. 8(b) shows that the increasing error at higher frequencies is largely due to a slight shift seen between the ROM and CFD results along with a slight amplitude discrepancy. Also, for the lift and moment coefficients at this particular test configuration, the corrected and uncorrected ROM results are very close to each other. In Figs. 7(a) (where the uncorrected ROM results are represented by dashed lines almost on top of the solid corrected ROM results) and 8(a), the two sets of results are virtually indistinguishable. As before, the drag results show a large discrepancy between the uncorrected ROM and corrected ROM/CFD results. 


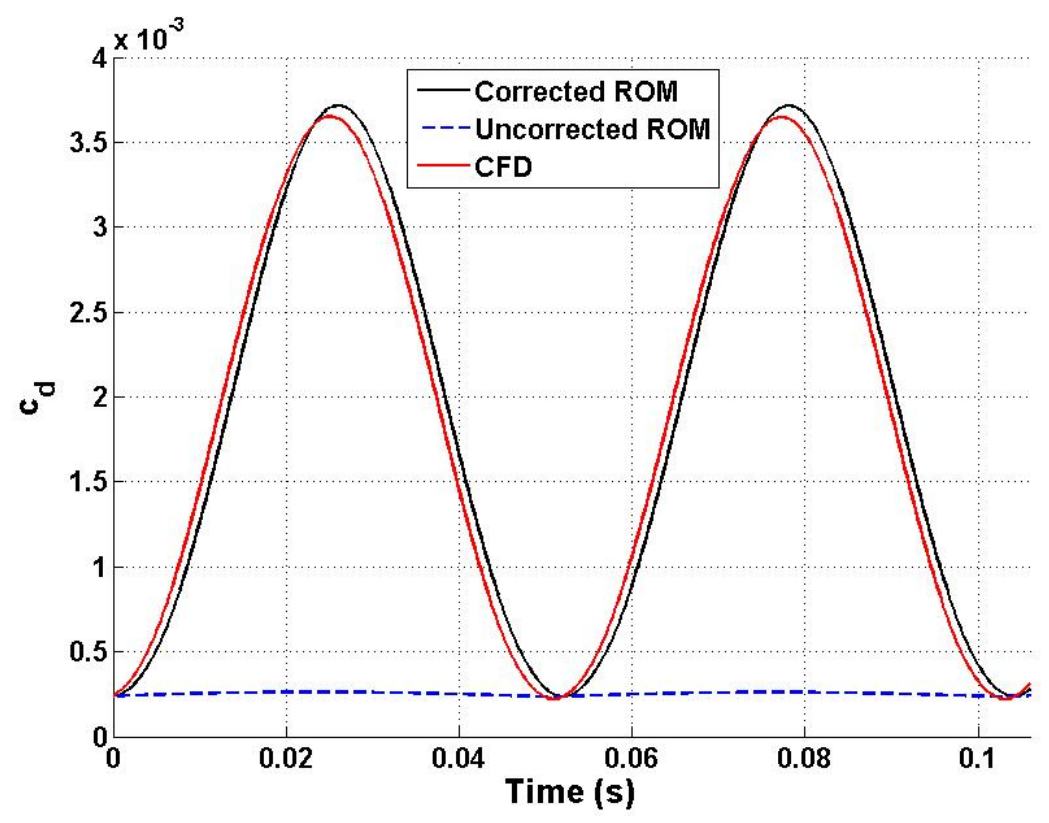

Figure 6. ROM-CFD comparison, Mach 0.9, amplitude 100×



(a) Mach 0.9

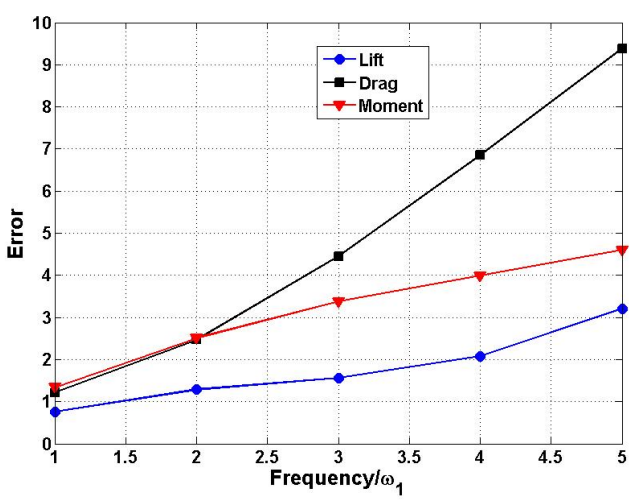

(b) Mach 1.1

Figure 7. Errors for increasing oscillation frequency 


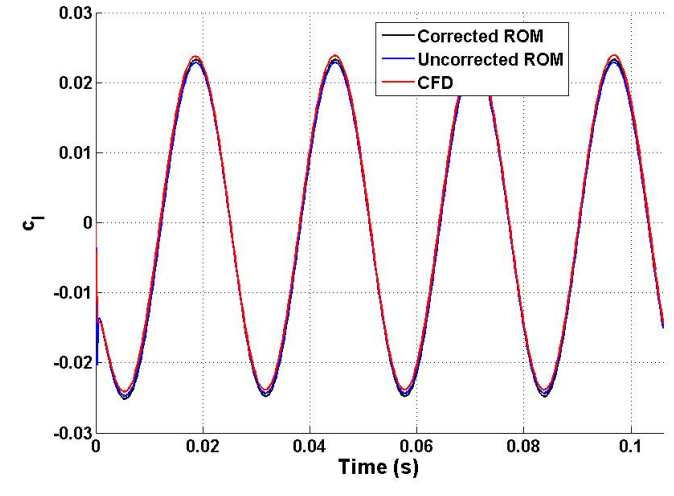

(a) Lift coefficient

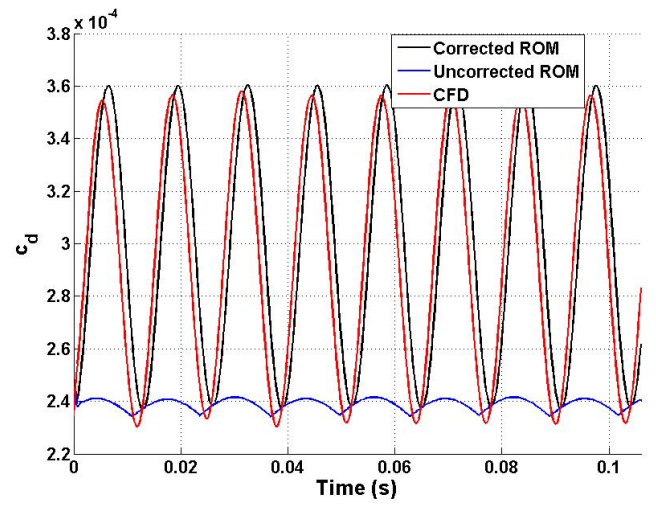

(b) Drag coefficient

Figure 8. ROM-CFD comparisons, Mach 0.9, $\omega=4 \omega_{1}$

\section{Multi-modal Oscillations}

Tests were conducted to investigate the accuracy of the ROM methodology when applied to multi-modal oscillations. For these tests, oscillations of the first three wing mode shapes were input into the CFD grid. As before, separate kriging surfaces were constructed for the subsonic and supersonic portions of the parameter space. Table 3 shows the ranges for each of the parameters considered, where $d_{i}$ is the amplitude of the $i^{t h}$ mode.

Table 3. Parameter ranges for multi-modal test cases

\begin{tabular}{ccc}
\hline \hline Parameter & Min & Max \\
\hline$M$ & 0.9 & 1.1 \\
$d_{1}$ & $-60 \times$ & $60 \times$ \\
$d_{2}$ & $-40 \times$ & $40 \times$ \\
$d_{3}$ & $-40 \times$ & $40 \times$ \\
\hline \hline
\end{tabular}

The initial kriging surfaces consisted of 39 sampling points, determined by Latin hypercube sampling, in each of the subsonic and supersonic regions. This number was chosen to provide a starting point in the determination as to the optimal number of total points that would be necessary. ROM and CFD results were generated for a sinusoidal test case, denoted as "Case 1," conducted with the parameters shown in Table 4. Fig. 9 shows the qualitative comparison between the various results. In that figure, "ROM1" represents the ROM constructed with the 39 sampling points mention previously. However, to see how sensitive the results would be to additional, strategically placed sampling points, an additional sampling point was placed at the location in the parameter space corresponding to the parameters at $t=0$; this is the result labelled "ROM2." As can be seen, placing the additional sampling point greatly improves the ROM-CFD agreement in that area. This suggests that, given enough sampling points in the correct locations, the ROM's accuracy could be greatly improved. The next section details efforts made to determine the amount and location of these additional points.

\section{A. Method of Segments}

The method of segments was implemented for this three-mode oscillation situation with a specific focus on the Mach number range from 1.0 to 1.1. Latin hypercube sampling was used to pick 22 steady runs at various angles of attack and Mach number. The range of angle of attack was chosen based on the maximum localized angle of attack expected to be seen based on the modal deformation ranges from Table 3. Kriging 
Table 4. Case 1 parameters

\begin{tabular}{cc}
\hline \hline$M$ & 0.96 \\
$d_{1}$ & $25 \times$ \\
$d_{2}$ & $12 \times$ \\
$d_{3}$ & $-10 \times$ \\
$\omega_{1}$ & $9.6 \mathrm{~Hz}$ \\
$\omega_{2}$ & $38.2 \mathrm{~Hz}$ \\
$\omega_{3}$ & $48.2 \mathrm{~Hz}$ \\
\hline \hline
\end{tabular}

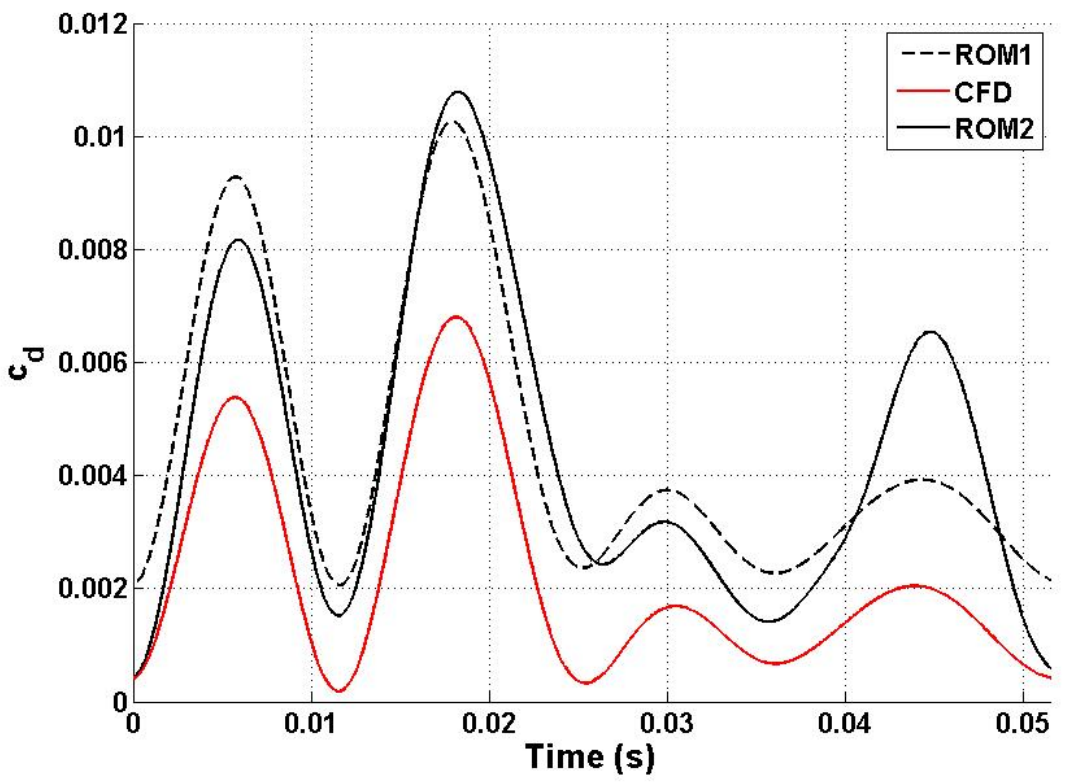

Figure 9. Case 1 drag comparison 
surfaces were constructed for each of the lift and drag forces at each segment along the wing span. Fig 10 shows sample surfaces for the segments along the root and tip.

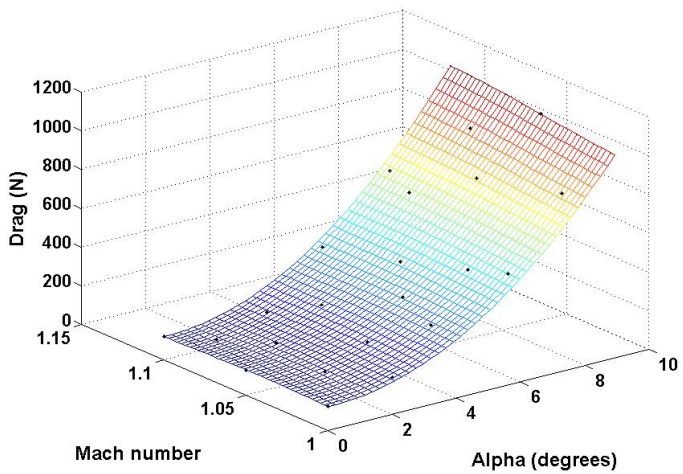

(a) Segment at root of wing



(b) Segment at tip of wing

Figure 10. Drag kriging surfaces for segments along wing span

To assess the accuracy of this method, the lift and drag coefficients obtained by CFD for each of the 39 multi-modal correction factor kriging surface runs were compared to coefficients obtained using the method of segments for the same parameters. Using the same root mean squared error metric as before, the lift coefficient error was $4.70 \%$, and the drag coefficient error was $5.61 \%$ over all test runs. This is shown graphically by Fig. 11 .

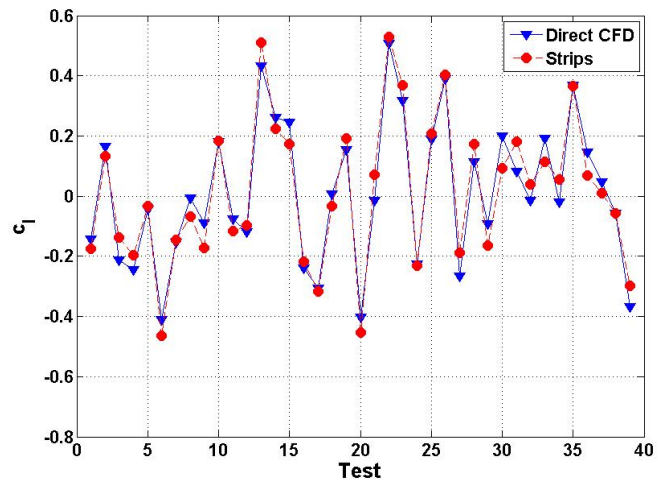

(a) Lift coefficients

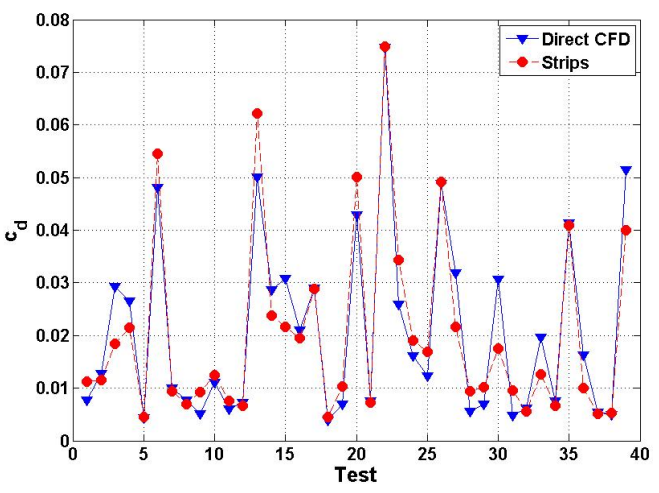

(b) Drag coefficients

Figure 11. Comparison of coefficients calculated by CFD and method of segments

Next, the method of segments was used to determine and then calculate the optimal number of sampling points for the parameter space consisting of three modes of oscillation and Mach number, again focusing on the range $M=1.0-1.1$. Using the 39 sampling points calculated with CFD, the maximum value of the MSEvariance ratio was 0.0012 for the drag correction factor surface. Employing the methodology of segments and choosing a MSE-variance ratio of 0.0010 as the "optimal" stopping point, a total of 118 sampling points were used in the parameter space. Note that picking a ratio of 0.0010 as optimal is arbitrary in this case, but the user can define the optimal stopping point based on the specific problem being considered. Fig. 12 shows the comparisons between the ROM constructed using only the 39 sampling points obtained directly from CFD (labelled as "ROM A"), the ROM constructed using the 118 sampling points from the method of segments ("ROM B"), and the direct CFD simulation. The parameters for the run, denoted as "Case 2," are found in Table 5. While the two ROMs give similar results for the lift coefficient, the additional points 
from the method of segments provide a significant improvement over the original ROM; the error decreases from $26.8 \%$ to $5.1 \%$.

Unlike the ROM constructed with direct CFD simulations for kriging surface sampling points, the method of segments does not suffer from the "curse of dimensionality." Unlimited sampling points could be calculated in the parameter space without conducting any more CFD runs, which allows the method of segments to be much more computationally efficient than a 1-to-1 CFD run to sampling point ratio. Also, the addition of more modes would not require any more CFD runs either. Because of this computational efficiency, the method of segments was shown to be an effective tool for the determination of parameter space sampling points.

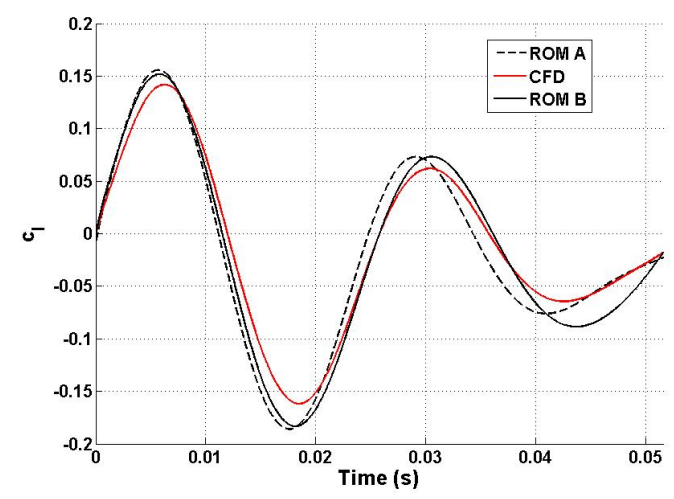

(a) Lift coefficient

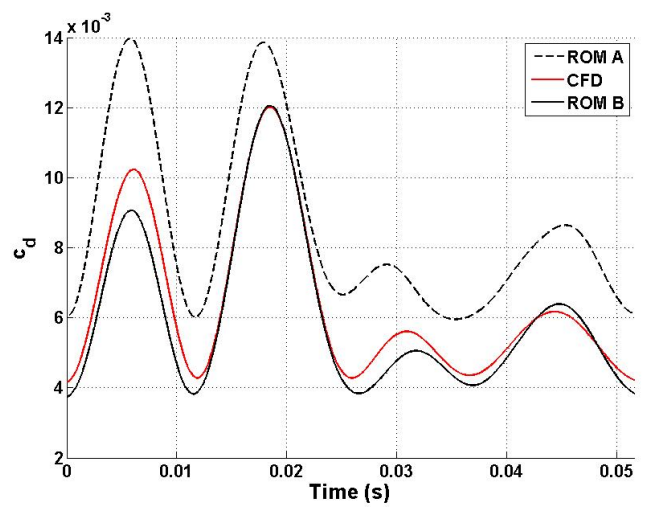

(b) Drag coefficient

Figure 12. ROM-CFD comparisons for Case 2

Table 5. Case 2 parameters

\begin{tabular}{cc}
\hline \hline$M$ & 0.96 \\
$d_{1}$ & $25 \times$ \\
$d_{2}$ & $12 \times$ \\
$d_{3}$ & $-10 \times$ \\
$\omega_{1}$ & $9.6 \mathrm{~Hz}$ \\
$\omega_{2}$ & $38.2 \mathrm{~Hz}$ \\
$\omega_{3}$ & $48.2 \mathrm{~Hz}$ \\
\hline \hline
\end{tabular}

\section{Concluding Remarks}

An unsteady reduced-order modeling methodology based on convolution combined with a nonlinear correction factor has been extended from the hypersonic regime to the transonic regime. Errors incurred through the use of the ROM have been assessed in terms of amplitude of oscillation as well as modal oscillation frequency. Additionally, a methodology for determining the optimal number of sampling points for ROM construction has been presented. The major conclusions are as follows:

- For single modal oscillations, errors remain small as amplitude of oscillation increases.

- ROM errors do increase with increased oscillation frequency. While the lift and moment errors remained relatively small over the range tested, the drag errors did show a larger increase. This increase was due to a slight phase shift between the ROM and CFD results which appeared at higher oscillation frequencies for the drag coefficient. 
- For a three-modal oscillation case, the method of segments was used to calculate the lift and drag coefficients for correction factor kriging surface construction. Over the 39 test points calculated both by the method of segments and direct CFD simulation, the root mean squared error between the two sets of results was just around $5 \%$ for each coefficient. Additionally, the methodology was applied to calculate additional sampling points to get the mean squared error of the kriging surface below a user-defined stopping criterion. In all, 118 sampling points were calculated by conducting only 22 steady CFD runs. The addition of further surface sampling points would not require any further CFD runs.

Future work will continue to investigate the applicability of the method of segments to the prediction of sampling point number and location in the parameter space. Also, this and other methods to reduce the overall number of CFD tests needed for kriging surface construction will be considered. Also, methods to reduce the errors at higher oscillation frequencies will be investigated.

\section{Acknowledgments}

Funds for the Michigan-AFRL Collaborative Center in Control Science (MACCCS) were made available from the Air Force Research Laboratory/Air Vehicles Directorate grant number FA 8650-07-2-3744. The program manager is Dr. Michael Bolender. The authors would also like to thank Dr. Walter Silva, Dr. Robert Bartels, and Dr. Robert Biedron from NASA Langley for their assistance in the computational studies.

\section{References}

${ }^{1}$ Silva, W., "Discrete-Time Linear and Nonlinear Aerodynamic Impulse Responses for Efficient CFD Analyses," Ph.D. dissertation, College of William \& Mary, December 1997.

${ }^{2}$ Silva, W., "Simultaneous Excitation of Multiple-Input/Multiple-Output CFD-Based Unsteady Aerodynamic Systems," Journal of Aircraft, Vol. 45, No. 4, July-August 2008, pp. 1267-1274.

${ }^{3}$ Raveh, D., Levy, Y., and Karpel, M., "Efficient Aeroelastic Analysis Using Computational Unsteady Aerodynamics," Journal of Aircraft, Vol. 38, No. 3, May-June 2001, pp. 547-556.

${ }^{4}$ Glaz, B., Liu, L., Friedmann, P., Bain, J., and Sankar, L., "A Surrogate Based Approach to Reduced-Order Dynamic Stall Modeling," Proceedings of the 51st AIAA/ASME/ASCE/AHS/ASC Structures, Structural Dynamics, and Materials Conference, AIAA Paper No. 2010-3042, April 2010.

${ }^{5}$ Silva, W., "Recent Enhancements to the Development of CFD-Based Aeroelastic Reduced-Order Models," Proceedings of the 48th AIAA/ASME/ASCE/AHS/ASC Structures, Structural Dynamics, and Materials Conference, AIAA Paper No. 2007-2051, April 2007.

${ }^{6}$ Lind, R., Prazenica, R., Brenner, M., and Baldelli, D., "Identifying Parameter-Dependent Volterra Kernels to Predict Aeroelastic Instabilities," AIAA Journal, Vol. 43, No. 12, December 2005, pp. 2496-2502.

${ }^{7}$ Baldelli, D., Zeng, J., Lind, R., and Harris, C., "Flutter-Prediction Tool for Flight-Test-Based Aeroelastic ParameterVarying Models," Journal of Guidance, Control, and Dynamics, Vol. 32, No. 1, January-February 2009, pp. $158-171$.

${ }^{8}$ Prazenica, R., Reisenthel, P., Kurdila, A., and Brenner, M., "Volterra Kernel Extrapolation for Modeling Nonlinear Aeroelastic Systems at Novel Flight Conditions," Journal of Aircraft, Vol. 44, No. 1, January-February 2007, pp. 149-162.

${ }^{9}$ Omran, A. and Newman, B., "Piecewise Global Volterra Nonlinear Modeling and Characterization for Aircraft Dynamics," Journal of Guidance, Control, and Dynamics, Vol. 32, No. 3, May-June 2009, pp. 749-759.

${ }^{10}$ Skujins, T. and Cesnik, C., "Reduced-Order Modeling of Hypersonic Vehicle Unsteady Aerodynamics," Proceedings of the AIAA Atmospheric Flight Mechanics Conference, AIAA Paper No. 2010-8127, August 2010.

${ }^{11}$ Fung, Y., An Introduction to the Theory of Aeroelasticity, Dover Publications, Mineola, New York, 1993.

${ }^{12}$ Cioppa, T. and Lucas, T., "Efficient Nearly Orthogonal and Space-Filling Latin Hypercubes," Technometrics, Vol. 49, No. 1, February 2007, pp. 45-55.

${ }^{13}$ Lophaven, S., Nielsen, H., and Sondergaard, J., "DACE: A MATLAB Kriging Toolbox, Version 2.0," Tech. Rep. IMMTR-2002-12, Informatics and Mathematical Modelling, Denmark, August 1, 2002.

${ }^{14}$ Sacks, J., Welch, W., Mitchell, T., and Wynn, H., "Design and Analysis of Computer Experiments," Statistical Science, Vol. 4, No. 4, 1989, pp. 409-435.

${ }^{15}$ Rumsey, C. and Biedron, R., "CFL3D User's Manual, Version 5.0, Second Edition," Tech. Rep. Hampton, VA, NASA Langley Research Center, September 1997 (last updated August 2009).

${ }^{16}$ Jr., E. Y., "AGARD Standard Aeroelastic Configurations for Dynamic Response. Candidate Configuration 1.-wing 445.6," Tech. Rep. NASA TM-100492, NASA Langley Research Center, 1987.

${ }^{17}$ Raveh, D., "Reduced-Order Models for Nonlinear Unsteady Aerodynamics," AIAA Journal, Vol. 39, No. 8, August 2001, pp. $1417-1429$.

${ }^{18}$ Raveh, D., "Identification of Computational-Fluid-Dynamics Based Unsteady Aerodynamic Models for Aeroelastic Analysis," Journal of Aircraft, Vol. 41, No. 3, May-June 2004, pp. 620-632.

${ }^{19}$ Cowan, T., A.S. Arena, J., and Gupta, K., "Accelerating Computational Fluid Dynamics Based Aeroelastic Predictions Using System Identification," Journal of Aircraft, Vol. 38, No. 1, January-February 2001, pp. 81-87. 
${ }^{20}$ Munteanu, S., Rajadas, J., and Chattopadhyay, A., "Nonlinear Aeroelastic Analysis Using a CFD-Based State Space ROM," Proceedings of the 47th AIAA/ASME/ASCE/AHS/ASC Structures, Structural Dynamics, and Materials Conference, AIAA Paper No. 2006-2024, May 2006.

${ }^{21}$ Silva, W. and Raveh, D., "Development of Unsteady Aerodynamic State-Space Models from CFD-Based Pulse Responses," Proceedings of the 42nd AIAA/ASME/ASCE/AHS/ASC Structures, Structural Dynamics, and Materials Conference and Exhibit, AIAA Paper No. 2001-25027, April 2001.

${ }^{22}$ Raveh, D., Levy, Y., and Karpel, M., "Aircraft Aeroelastic Analysis and Design Using CFD-Based Unsteady Loads," Proceedings of the 41st AIAA/ASME/ASCE/AHS/ASC Structures, Structural Dynamics, and Materials Conference and Exhibit, AIAA Paper No. 2000-24645, April 2000.

${ }^{23}$ Jones, R., Schonlau, M., and Welch, W., "Efficient Global Optimization of Expensive Black-Box Functions," Journal of Global Optimization, Vol. 13, No. 4, 1998, pp. 455-492. 